This item was submitted to Loughborough's Research Repository by the author.

Items in Figshare are protected by copyright, with all rights reserved, unless otherwise indicated.

\title{
Hierarchical modeling and speed control of networked induction motor systems
}

PLEASE CITE THE PUBLISHED VERSION

https://doi.org/10.1109/ACC.2013.6580184

PUBLISHER

IEEE (@ American Automatic Control Council)

VERSION

VoR (Version of Record)

\section{PUBLISHER STATEMENT}

This work is made available according to the conditions of the Creative Commons Attribution-NonCommercialNoDerivatives 4.0 International (CC BY-NC-ND 4.0) licence. Full details of this licence are available at: https://creativecommons.org/licenses/by-nc-nd/4.0/

\section{LICENCE}

CC BY-NC-ND 4.0

\section{REPOSITORY RECORD}

Zhao, Dezong, Chunwen Li, and Richard Stobart. 2017. "Hierarchical Modeling and Speed Control of Networked Induction Motor Systems". figshare. https://hdl.handle.net/2134/26518. 


\title{
Hierarchical Modeling and Speed Control of Networked Induction Motor Systems*
}

\author{
Dezong Zhao ${ }^{1}$, Member, IEEE, Chunwen $\mathrm{Li}^{2}$, and Richard Stobart ${ }^{1}$, Member, IEEE
}

\begin{abstract}
This paper proposes a hierarchical modeling method and a fuzzy speed control strategy for nonlinear networked induction motor systems subject to network induced time delay and packets dropout. The networked induction motor control system consists of a networked speed controller and a local controller. Fuzzy gain scheduling is applied on the networked speed controller to guarantee the robustness against complicated variations on the communication network. The state predictor is to compensate the time delay occurred in data transmission in the feedback channel. In stability analysis, the upper allowed limits of the time delay and packets dropout are calculated using the Lyapunov-Krasovskii theorem, respectively. Simulation and experimental results are given to illustrate the effectiveness of the proposed approach.
\end{abstract}

\section{INTRODUCTION}

The applications of NCSs have been an important trend in modern industry owing to the convenient remote operation and cost-effective installation. In such systems, spatially distributed sensors, actuators, and controllers share information through the network instead of complex wiring, resulting in flexible and open architecture. NCSs have been found applications in a broad range of areas such as mobile robots [1]-[3], unmanned aerial vehicles [4]-[6], and remote surgery [7]-[9]. Considering the common grounds that they are driven by electrical motors and communicate via network, such systems are called networked motion control systems (NMCSs) [10]. NMCSs are constructed on the basis of remote motion controller and local motor drivers, using network to realize transmission of control orders and motion states. NMCSs are hot research topics of NCSs and play important roles in factory automation.

Most of the current NMCSs focus on networked DC motor control [11]-[13], for DC motor is an ideal networked control plant with linear model. Actually, induction motors play a dominant part in industrial applications for their merits of simple structure and high reliability. However, networked induction motor control is rather more complicated due to the nonlinear dynamics of induction motors. In this paper, the modeling and speed control of nonlinear networked induction motor control systems are investigated. Using field orientation technique, the induction motor model is linearized, and the NMCS is built as a hierarchical control system, with the merit of free of complicated modeling and data processing.

\footnotetext{
*This work was partially supported by National Natural Science Foundation of China under the grant reference 61174068

${ }^{1}$ D. Zhao and R. Stobart are with Department of Aeronautical and Automotive Engineering, Loughborough University, Loughborough, Leicester, UK (e-mail: d.zhao2@lboro.ac.uk,r.k.stobart@lboro.ac.uk)

${ }^{2} \mathrm{C}$. Li is with Department of Automation, Tsinghua University, Beijing, China (e-mail: lcw@mail.tsinghua.edu.cn)
}

Fuzzy logic is applied on gain adaptation in designing the networked speed controller, such that the variation of the network QoS can be compensated. Considering the time delay from sensor to controller can be seized owing to the time stamped message, a time delay compensator is designed in the feedback channel. As an indispensable part, the stability of the controlled system is analyzed using Lyapunov-Krasovskii theorem, with the maximum allowed bounds of time delay and packets dropout are provided.

The paper is organized as following. After the introduction in section I, the system description is presented in section II. The networked speed controller is proposed in section III. The stability analysis is presented in section IV. The simulation and experimental results are stated in section V. Finally, the conclusions are summarized in section VI.

\section{System Description}

As shown in Fig. 1, the components of the considered NMCS can be grouped into five modules, which are described in the following subsections, respectively.

\section{A. Induction Motor and the Sensor}

The dynamics of a three-phase squirrel induction motor in the stator fixed $\alpha-\beta$ reference frame is described as the following differential equations [14]:

$$
\begin{aligned}
& \dot{i}_{\alpha s}=-\gamma i_{\alpha s}+\alpha \beta \psi_{\alpha r}+n_{p} \beta \omega \psi_{\beta r}+u_{\alpha s} /\left(\sigma L_{s}\right), \\
& \dot{i}_{\beta s}=-\gamma i_{\beta s}+\alpha \beta \psi_{\beta r}-n_{p} \beta \omega \psi_{\alpha r}+u_{\beta s} /\left(\sigma L_{s}\right), \\
& \dot{\psi}_{\alpha r}=\alpha M i_{\alpha s}-\alpha \psi_{\alpha r}-n_{p} \omega \psi_{\beta r}, \\
& \dot{\psi}_{\beta r}=\alpha M i_{\beta s}-\alpha \psi_{\beta r}+n_{p} \omega \psi_{\alpha r}, \\
& \dot{\omega}=\mu\left(\psi_{\alpha r} i_{\beta s}-\psi_{\beta r} i_{\alpha s}\right)-\left(T_{L}+K_{f} \omega\right) / J .
\end{aligned}
$$

where the two-dimensional vectors $i_{s}=\left[\begin{array}{ll}i_{\alpha s} & i_{\beta s}\end{array}\right]^{\mathrm{T}}, \psi_{r}=$ $\left[\begin{array}{ll}\psi_{\alpha r} & \psi_{\beta r}\end{array}\right]^{\mathrm{T}}$, and $u=\left[\begin{array}{ll}u_{\alpha s} & u_{\beta s}\end{array}\right]^{\mathrm{T}}$ are the stator currents, rotor fluxes, and stator voltages, respectively. $\omega$ is the

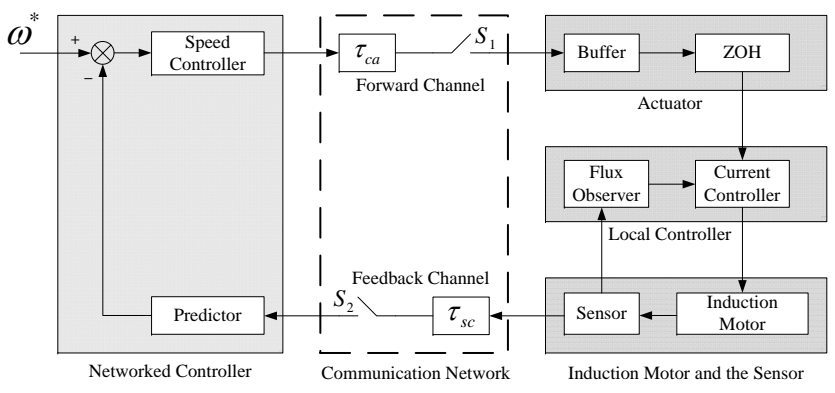

Fig. 1. Structure of the investigated NMCS 


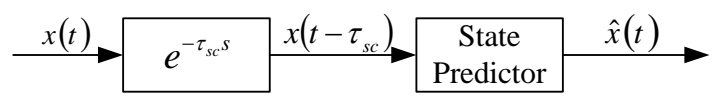

Fig. 2. State flow of the predictor

mechanical rotor speed, $R_{S}$ and $R_{r}$ are the stator and rotor resistances, respectively; $L_{s}$ and $L_{r}$ are the stator and rotor self-inductances, respectively; $M$ is the stator-rotor mutual inductance, $T_{L}$ is the load torque, $K_{f}$ is the friction coefficient, $J$ is the motor-load moment of inertia, and $n_{p}$ is the number of pole pairs. Denote the leakage factor by $\sigma=1-M^{2} /\left(L_{s} L_{r}\right)$, the rotor time constant by $T_{r}=L_{r} / R_{r}$, and the other parameters by $\alpha=1 / T_{r}, \beta=M /\left(\sigma L_{s} L_{r}\right)$, $\gamma=M^{2} R_{r} /\left(\sigma L_{s} L_{r}^{2}\right)+R_{s} /\left(\sigma L_{s}\right)$, and $\mu=3 n_{p} M /\left(2 J L_{r}\right)$. The mechanical equation (1e) can be expressed in terms of the electromagnetic torque $T_{e}$ :

$$
T_{e}=J \dot{\omega}+K_{f} \omega+T_{L} .
$$

The induction motor speed is measured by the sensor periodically, and be sent to the networked controller via the network together with its time stamp.

\section{B. Communication Network}

The network-induced delay consists of the sensor-tocontroller delay $\tau_{s c}$ and the controller-to-actuator delay $\tau_{c a}$, and can be lumped together as $\tau=\tau_{s c}+\tau_{c a}$. Two switches $S_{1}$ and $S_{2}$ are used to model the packets dropout in the forward and feedback channels of the network.

\section{Networked Controller}

The networked controller consists of two parts: the speed controller and the state predictor. A fuzzy logic PI controller is employed as the speed controller, where the gain values are tuned online by the fuzzy logic mechanism. The state predictor estimates the motion state $\hat{x}(t)$ based on the plant state $x(t)$ and the feedback delay $\tau_{s c}$, as shown in Fig. 2 .

\section{Actuator}

The actuator is triggered when receiving data from the controller. The buffer size of the actuator is 1, to guarantee the latest control packet is used. Any newly arrived control packet at the actuator will update the control signal with older time stamp (if existing) in the buffer, otherwise, it will be discarded. At each sampling instant, the control command in the buffer is read by the zero-order hold $(\mathrm{ZOH})$ circuit and sent to the motor.

\section{E. Local Controller}

The local controller consists of the current regulator and the flux observer, to implement the inner loop torque control, which is assumed fast enough in tracking the commands of the stator currents. Is is reasonable with power electronics are available today. A sliding mode estimator and a PI controller are adopted as the flux observer and current regulator, respectively. The readers can refer to [15] for more details and the references therein. Using field orientation technique, the induction motor model is simplified as a DC motor linear model. The synchronous rotating angle of the rotor flux can be calculated from the estimated flux:

$$
\hat{\theta}_{e}=\arctan \left(\hat{\psi}_{\beta r} / \hat{\psi}_{\alpha r}\right) .
$$

The stator currents under the synchronous rotating $d-q$ coordination are obtained by

$$
\left[\begin{array}{l}
i_{d s} \\
i_{q s}
\end{array}\right]=\left[\begin{array}{cc}
\cos \left(\hat{\theta}_{e}\right) & \sin \left(\hat{\theta}_{e}\right) \\
-\sin \left(\hat{\theta}_{e}\right) & \cos \left(\hat{\theta}_{e}\right)
\end{array}\right]\left[\begin{array}{l}
i_{\alpha s} \\
i_{\beta s}
\end{array}\right],
$$

and the rotor fluxes $\hat{\psi}_{q r}=0$ and $\hat{\psi}_{d r}=\sqrt{\hat{\psi}_{\alpha r}^{2}+\hat{\psi}_{\beta r}^{2}}$ are satisfied under rotor field orientation. Accordingly, the mechanical equation (1e) can be represented as

$$
\dot{\omega}=\frac{K_{t}}{J} i_{q s}-\frac{K_{f}}{J} \omega-\frac{T_{L}}{J},
$$

where $K_{t}=\mu \hat{\psi}_{d r}$.

\section{Networked Controller Design}

Considering the influence of the QoS variation on the control performance of the induction motor, fuzzy logic is adopted in gain adaptation of the networked speed controller. Furthermore, the state predictor placed in the feedback channel is employed to minimize the trajectory deviation due to the time delay.

\section{A. Speed Controller Design}

The fuzzy control rules are defined in Table I. $K_{P}$ and $K_{I}$ are initialized according to the no-delay system and are tuned online by the fuzzy inference according to the feedback speed error, such that the control command is updated to compensate the delay. The updating law of the gains are

$$
\begin{aligned}
K_{P}^{\prime} & =K_{P}+\Delta K_{P} \\
K_{I}^{\prime} & =K_{I}+\Delta K_{I}
\end{aligned},
$$

where $\Delta K_{P}$ and $\Delta K_{I}$ are the increment values of $K_{P}$ and $K_{I}$, respectively, while $K_{P}^{\prime}$ and $K_{I}^{\prime}$ are the updated gains.

\section{B. State Predictor Design}

The state predictor is based on the feedback data measured by the speed sensor. $\tau_{s c}$ and $\tau_{c a}$ are different in nature where $\tau_{s c}$ can be known when the controller uses the sensor data to generate the control signal, provided the sensor message is time stamped. Therefore, a predictor can be used to estimate the available plant state in calculating the control law. However, $\tau_{s c}$ can not be compensated using the time

TABLE I

FUZZY CONTROL RULE BASE

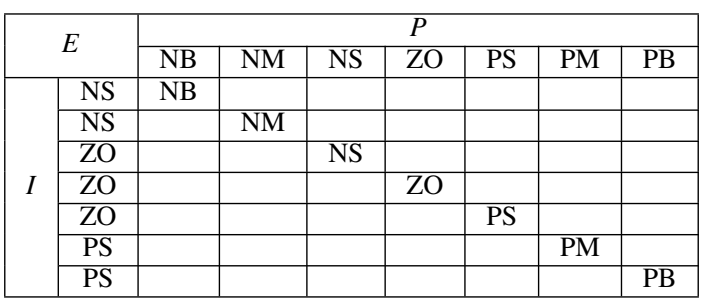




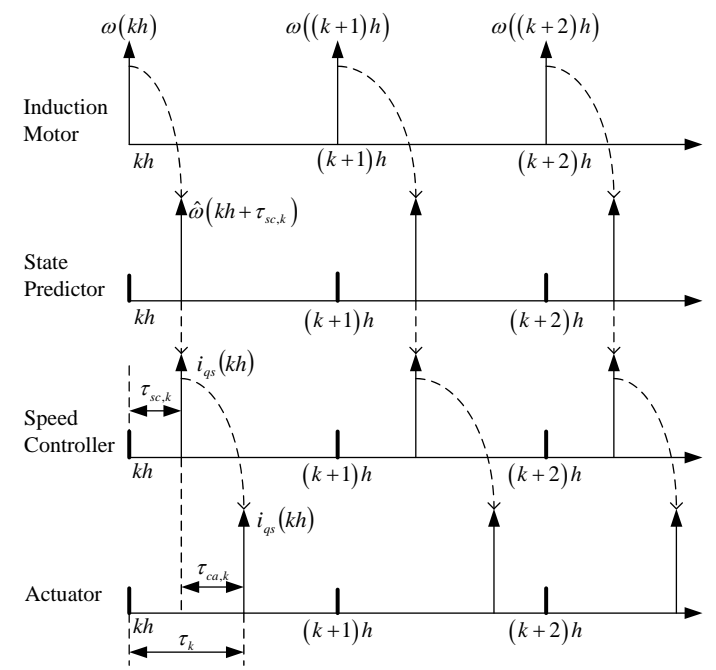

Fig. 3. Timing diagram of the signals in NMCS

stamp method in decision making of control laws. The state predictor is used to compensate $\tau_{s c}$, to obtain a more accurate plant state estimation. It is supposed a separated cooling fan is used, then $K_{f}$ can be neglected. Therefore, (5) can be expressed as

$$
\dot{\omega}=\frac{K_{t}}{J} i_{q s}-\frac{T_{L}}{J}
$$

therefore the motor speed can be obtained by

$$
\omega(t)=\omega\left(t_{0}\right)+\frac{K_{t}}{J} \int_{t_{0}}^{t} i_{q s}(s) \mathrm{d} s-\frac{T_{L}}{J}\left(t-t_{0}\right) .
$$

The timing diagram of the signals in NMCS considering the state prediction is shown as Fig. 3, where $\tau_{k}$ denotes the lumped time delay in the sampling period $[k h,(k+1) h]$, $\tau_{s c, k}$ denotes the sensor-to-controller delay for the sampled motor speed $\omega(k h), \tau_{c a, k}$ denotes the controller-to-actuator delay for the control command $i_{q s}(k h)$, and $\hat{\omega}\left(k h+\tau_{s c, k}\right)$ denotes the compensated feedback speed. The compensated speed signal within $[k h,(k+1) h]$ can be represented by the following discretized equation:

$$
\hat{\omega}\left(k h+\tau_{s c, k}\right)=\omega(k h)+\left(\frac{K_{t}}{J} i_{q s}(k h)-\frac{1}{J} T_{L}\right) \tau_{s c, k} .
$$

\section{Stability Analysis}

In this section, the influences of the time delay and the packets dropout on the stability of the NMCS are investigated. The time delay $\tau_{k}$ is assumed less than the sampling period $h$. The packets dropout rate satisfies $0 \leq r \leq 1$. The mechanical equation (5) can be written in form of the state space equation:

$$
\left\{\begin{array}{l}
\dot{\bar{x}}(t)=A \bar{x}(t)+B u(t)+E \\
y(t)=C \bar{x}(t)
\end{array}\right.
$$

where $\bar{x}(t)=\omega(t), u(t)=i_{q s}(t), E=-\frac{T_{L}}{J}$, and $y(t)=\omega(t)$, with the coefficients of $A=-\frac{K_{f}}{J}, B=\frac{K_{t}}{J}$, and $C=1$. Taking into account that (10a) can be represented as

$$
\frac{\mathrm{d}}{\mathrm{d} t}\left(\bar{x}+\frac{E}{A}\right)=A\left(\bar{x}+\frac{E}{A}\right)+B u(t),
$$

the closed loop NMCS model can be expressed as

$$
\left\{\begin{array}{l}
\dot{x}(t)=A x(t)+B u(t) \\
y(t)=C x(t)
\end{array}\right.
$$

in stability analysis for convenience.

\section{A. Stability Analysis with Time Delay}

The maximum allowed time delay bound for a stable system is independent of network protocols, denoted as $\bar{\tau}$. The Lyapunov-Krasovskii function (LKF) method is used to calculate $\bar{\tau}$. For $K_{I}$ is primarily used to eliminate the steady state error, the networked controller can be treated as a state feedback controller in stability analysis:

$$
u(t)=-K_{P} x(t-\tau)
$$

The closed-loop NMCS model (12) is represented as:

$$
\dot{x}(t)=A x(t)+M x(t-\tau),
$$

with $M=-B K_{P}$. Several criteria are introduced in analyzing the stability of NMCS:

Lemma 1: [16] Assume that $a(\cdot) \in \mathbb{R}^{n_{a}}, b(\cdot) \in \mathbb{R}^{n_{b}}$, and $W(\cdot) \in \mathbb{R}^{n_{a} \times n_{b}}$ are defined on the interval $\Omega$. For any matrices $X \in \mathbb{R}^{n_{s} \times n_{s}}, Y \in \mathbb{R}^{n_{s} \times n_{b}}$, and $Z \in \mathbb{R}^{n_{b} \times n_{b}}$ satisfying $\left[\begin{array}{cc}X & Y \\ Y^{T} & Z\end{array}\right] \geq 0$, the following inequality holds:

$$
\begin{aligned}
& -2 \int_{\Omega} a^{\mathrm{T}}(\alpha) W b(\alpha) \mathrm{d} \alpha \\
& \quad \leq \int_{\Omega}\left[\begin{array}{l}
a(\alpha) \\
b(\alpha)
\end{array}\right]^{\mathrm{T}}\left[\begin{array}{cc}
X & Y-W \\
Y^{\mathrm{T}}-W^{\mathrm{T}} & Z
\end{array}\right]\left[\begin{array}{l}
a(\alpha) \\
b(\alpha)
\end{array}\right] \mathrm{d} \alpha .
\end{aligned}
$$

The Schur complement lemma can be transformed into the form of Riccati inequality:

Lemma 2: [17] For the given constant matrices $\mathscr{A}$ and $\mathscr{Q}=\mathscr{Q}^{\mathrm{T}}$, if exists matrix variable $\mathscr{P}>0$ satisfying

$$
\left[\begin{array}{cc}
\mathscr{Q} & \mathscr{A} \\
\mathscr{A}^{\mathrm{T}} & -\mathscr{P}^{-1}
\end{array}\right]<0
$$

then the following inequality holds:

$$
\mathscr{A} \mathscr{P} \mathscr{A}^{\mathrm{T}}+\mathscr{Q}<0 \text {. }
$$

The following theorem represents the delay-dependent stability condition of the NMCS:

Theorem 1: If there exist matrices $P>0, Q>0$, and $X$, $Y, Z$ with appropriate dimensions such that

$$
\left[\begin{array}{ccc}
\Gamma & P M-Y & \bar{\tau} A Z \\
M^{\mathrm{T}} P^{\mathrm{T}}-Y^{\mathrm{T}} & -Q & \bar{\tau} M Z \\
\bar{\tau} Z^{\mathrm{T}} A^{\mathrm{T}} & \bar{\tau} Z^{\mathrm{T}} M^{\mathrm{T}} & -\bar{\tau} Z
\end{array}\right]<0
$$

and

$$
\left[\begin{array}{ll}
X & Y \\
Y^{\mathrm{T}} & Z
\end{array}\right] \geq 0
$$

where $\Gamma=A^{\mathrm{T}} P+P A+Y+Y^{\mathrm{T}}+Q+\bar{\tau} X$, then the system (14) is asymptotically stable for any time delay $0 \leq \tau \leq \bar{\tau}$.

Using Theorem 1, $\bar{\tau}$ can be obtained via the given networked speed controller gain $K_{P}$. 


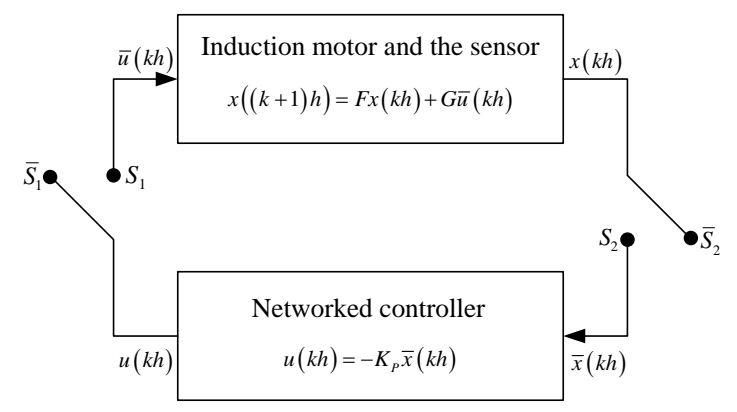

Fig. 4. Data transmission in NMCS with packets dropout

\section{B. Stability Analysis with Packets Dropout}

The discrete NMCS model is given as

$$
\left\{\begin{array}{l}
x((k+1) h)=F x(k h)+G u(k h) \\
y(k h)=C x(k h)
\end{array},\right.
$$

with $F=e^{A h}$ and $G=\int_{0}^{h} e^{A t} B d t$. The discrete networked controller is

$$
u(k h)=-K_{P} \hat{x}(k h)
$$

where $\hat{x}(k h) \triangleq x\left(k h-\tau_{k}\right)$.

The stability of the NMCS with packets dropout can be analyzed according to the criteria in the asynchronous dynamical system (ADS). Consider an ADS

$$
x(k+1)=f_{i}(x(k)), \quad i=1,2, \ldots, n .
$$

If existing $\alpha \geq 1$ such that

$$
\lim _{k \rightarrow \infty} \alpha^{k}\|x(k)\|=0,
$$

then the ADS (22) is called exponentially stable, and the upper allowed bound of $\alpha$ is referred to as the decay rate of the system. Hassibi et. al. proposed the stability condition of the ADS, which is described as the following theorem [18]:

Theorem 2: If there exist a Lyapunov function $V(x(k))$ : $\mathbb{R}^{n} \rightarrow \mathbb{R}_{+}$:

$$
\beta_{1}\|x\|^{2} \leq V(x) \leq \beta_{2}\|x\|^{2}
$$

and scalars $\alpha_{1}, \alpha_{2}, \ldots, \alpha_{n}$, with corresponding packets droping rate $r_{i}$ such that

$$
\alpha_{1}^{r_{1}} \alpha_{2}^{r_{2}} \cdots \alpha_{n}^{r_{n}}>\alpha>1
$$

and

$$
V(x(k+1))-V(x(k)) \leq\left(\alpha_{i}^{-2}-1\right) V(x(k)),
$$

then the ADS (22) remains exponentially stable with the decay rate greater than $\alpha$.

Fig. 4 illustrates the data transmission in NMCS with packets dropout in both the forward channel and feedback channel. The two switches $S_{1}$ and $S_{2}$ are used to simulate the packets dropout at a certain rate $r$. When the switches $S_{i}(i=$ $1,2)$ are closed, the packets are transmitted successfully, otherwise the packets are lost and the output of the switches are held as the previous value. Therefore the dynamics of the switches can be modeled as:

$$
\begin{aligned}
& S_{1}: \bar{u}(k h)=u(k h), \\
& \bar{S}_{1}: \bar{u}(k h)=u((k-1) h), \\
& S_{2}: \bar{x}(k h)=x(k h), \\
& \bar{S}_{2}: \bar{x}(k h)=x((k-1) h) .
\end{aligned}
$$

A new vector about the switches is introduced as

$$
S=\left[\begin{array}{ll}
\tilde{S}_{1} & \tilde{S}_{2}
\end{array}\right]
$$

where $\tilde{S}_{i}$ can be $S_{i}$ or $\bar{S}_{i},(i=1,2)$. Defining a new state vector in the NMCS as

$$
z(k h)=\left[\begin{array}{lll}
x(k h) & \bar{x}(k h) & \bar{u}(k h)
\end{array}\right]
$$

then the NMCS with packets dropout can be modeled as

$$
z((k+1) h)=\Theta_{i} z(k h),
$$

with $i=1,2,3,4$. Therefore, the inequality (25) can be rewritten as

$$
\alpha_{1}^{r} \alpha_{2}^{r} \alpha_{3}^{1-r} \alpha_{4}^{1-r}>\alpha>1
$$

Depending on the status of switches, $\Theta_{i}$ is represented as
1) $\Theta_{1}=\left[\begin{array}{ccc}F & 0 & G \\ 0 & I & 0 \\ 0 & 0 & I\end{array}\right]$, if $S=\left[\begin{array}{ll}\bar{S}_{1} & \bar{S}_{2}\end{array}\right]$;
2) $\Theta_{2}=\left[\begin{array}{ccc}F & 0 & G \\ F & 0 & G \\ 0 & -K_{P} & 0\end{array}\right]$, if $S=\left[\begin{array}{ll}S_{1} & S_{2}\end{array}\right]$;
3) $\Theta_{3}=\left[\begin{array}{ccc}F & 0 & G \\ 0 & I & 0 \\ 0 & -K_{P} & 0\end{array}\right]$, if $S=\left[\begin{array}{ll}S_{1} & \bar{S}_{2}\end{array}\right]$;
4) $\Theta_{4}=\left[\begin{array}{lll}F & 0 & G \\ F & 0 & G \\ 0 & 0 & I\end{array}\right]$, if $S=\left[\begin{array}{ll}\bar{S}_{1} & S_{2}\end{array}\right]$.

Choosing the Lyapunov function of NMCS (30) as

$$
V(z)=z^{\mathrm{T}} P z, \quad P>0,
$$

then finding the upper allowed bound of packets dropout $\bar{r}$ can be formulated as an optimization problem:

Maximize : $\quad \alpha_{1}^{r} \alpha_{2}^{r} \alpha_{3}^{1-r} \alpha_{4}^{1-r}$

$$
\begin{array}{ll}
\text { Subject to : } & \Theta_{1}^{\mathrm{T}} P \Theta_{1}-P \leq\left(\left(\alpha_{3} \alpha_{4}\right)^{-2}-1\right) P, \\
& \Theta_{2}^{\mathrm{T}} P \Theta_{2}-P \leq\left(\left(\alpha_{1} \alpha_{2}\right)^{-2}-1\right) P, \\
& \Theta_{3}^{\mathrm{T}} P \Theta_{3}-P \leq\left(\left(\alpha_{3} \alpha_{2}\right)^{-2}-1\right) P, \\
& \Theta_{4}^{\mathrm{T}} P \Theta_{4}-P \leq\left(\left(\alpha_{1} \alpha_{4}\right)^{-2}-1\right) P .
\end{array}
$$

The inequalities in (33) can be rewritten as bilinear matrix inequalities (BMI) in finding $P$ and $\alpha_{1}, \alpha_{2}, \alpha_{3}, \alpha_{4}$. Several methods such as genetic algorithms [19] can be used in solving (33). In the case of packets dropout, the control performance of a NMCS can be improved by reducing the sampling period.

\section{Simulation and Experimental Results}

In this section, the performance of the proposed fuzzy tuned gain scheduling control method and state predictor are verified in simulation and experiment. 
TABLE II

Parameters of the Simulated Induction Motor

\begin{tabular}{lll}
\hline Parameters & Values & Units \\
\hline$R_{r}$ & 5.5 & $\Omega$ \\
$R_{s}$ & 6.7 & $\Omega$ \\
$L_{r}$ & 0.475 & $\mathrm{H}$ \\
$L_{s}$ & 0.475 & $\mathrm{H}$ \\
$M$ & 0.45 & $\mathrm{H}$ \\
$J$ & 0.015 & $\mathrm{kgm}^{2}$ \\
$n_{p}$ & 2 & - \\
\hline
\end{tabular}

TABLE III

RATED PARAMETERS OF THE EXPERIMENTAL INDUCTION MOTOR

\begin{tabular}{lll}
\hline Parameters & Values & Units \\
\hline$P_{N}$ & 0.287 & $\mathrm{~kW}$ \\
$V_{N}$ & $220 / 380$ & $\mathrm{~V}$ \\
$f_{N}$ & 50 & $\mathrm{~Hz}$ \\
$I_{N}$ & $2.69 / 1.55$ & $\mathrm{~A}$ \\
$n_{N}$ & 1400 & $\mathrm{rpm}$ \\
\hline
\end{tabular}

\section{A. Simulation Results}

Simulations of the NMCS are carried out on the Matlab/Simulink platform using the Truetime toolbox. The network protocol is selected as CSMA/AMP (CAN), with the data rate of $10^{5} \mathrm{bits} / \mathrm{s}$, and the minimum frame size of 64 bits. The parameters of the induction motor is shown in Table II, and the reference speed and reference rotor flux are selected as $\omega^{*}=100 \mathrm{rad} / \mathrm{s}$ and $\psi_{d r}^{*}=1 \mathrm{~Wb}$, respectively. It is supposed that $\tau_{s c}=\tau_{c a}=\tau / 2$.

Substituting $K_{P 0}$ into Theorem $1, \bar{\tau}=0.04 \mathrm{~s}$ is obtained using the functions in Matlab linear matrix inequalities (LMI) toolbox. The sampling period is selected as $h=0.01 \mathrm{~s}$, and the corresponding $\bar{r}$ is obtained as $84.6 \%$ via Theorem 2 .

The performance of the networked speed controller at time-varying $\tau$ is depicted in Fig. 5. As a comparison, the control performance of using the traditional PI controller and the memoryless state feedback controller ( $\mathrm{P}$ controller) are both given. The simulation results illustrate that the fuzzy PI controller behaves better than the other two methods. The P controller holds the slowest transient performance and largest steady state error, for the least feedback information has used. The PI controller improves the steady state error, but the transient performance is still far from acceptable, because of the initial values of $K_{P}$ and $K_{I}$ are set manually. The proposed fuzzy PI controller has the best transient performance, due to $K_{P}$ and $K_{I}$ are tuned online, to adapt variations on the network QoS.

The state predictor behaves as an important part in improving the feedback information. Its performance at constant delay and time-varying delay are evaluated in Fig. 6. The performance of the networked controller without state predictor and without time delay are also plotted. It is clear that the NMCS has the best tracking performance under ideal network circumstance. The controller with state predictor holds better tracking performance than that without state predictor under both of the two test conditions, for the feedback delay $\tau_{s c}$ is compensated by the state predictor.

The control performance of the NMCS with packets dropout is shown in Fig. 7, while the packets dropout rate is set as $r=30 \%$. Using the conclusion in stability analysis with packets dropout, the control performance can be improved by reducing the sampling period. Therefore, by resetting the sampling period as $h=0.007 \mathrm{~s}$ and $h=0.002 \mathrm{~s}$, respectively, the corresponding speed curves illustrate that the NMCS performance is significantly improved.

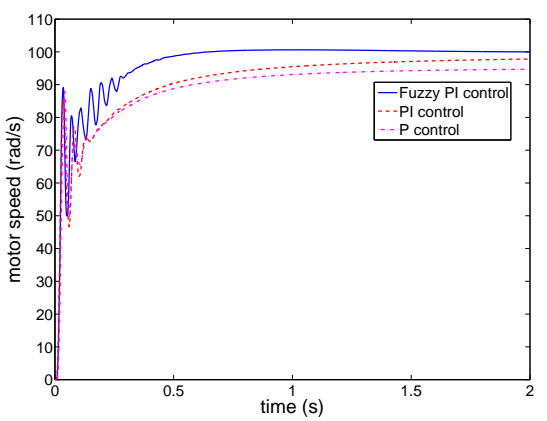

Fig. 5. Control performance evaluation with $5 \mathrm{~ms} \leq \tau \leq 20 \mathrm{~ms}$

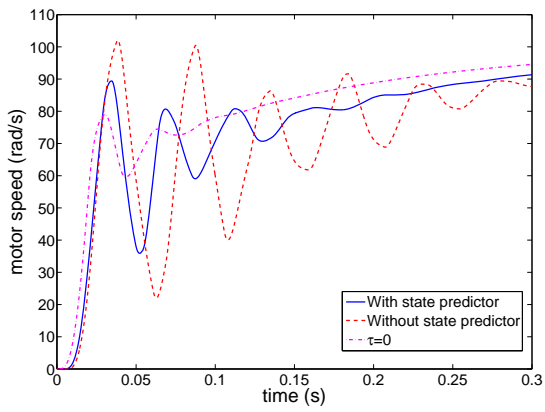

Fig. 6. State predictor performance evaluation with $5 \mathrm{~ms} \leq \tau \leq 20 \mathrm{~ms}$

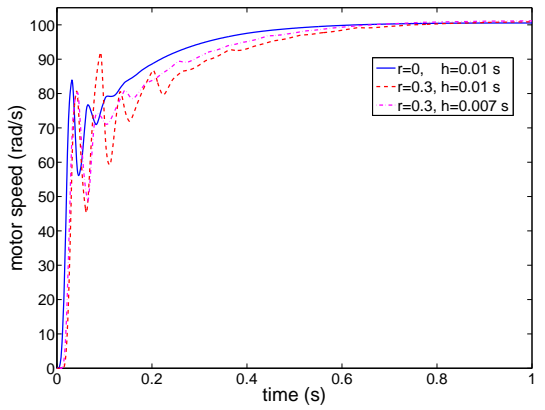

Fig. 7. Control performance evaluation with packets dropout

\section{B. Experimental Results}

To verify the proposed control strategy, a NMCS experiment platform is built, whose structure is shown as Fig. 8 . The local control system consists of the local control module, drive module, induction motor, and encoder; the networked control module behaves as the networked speed controller. 


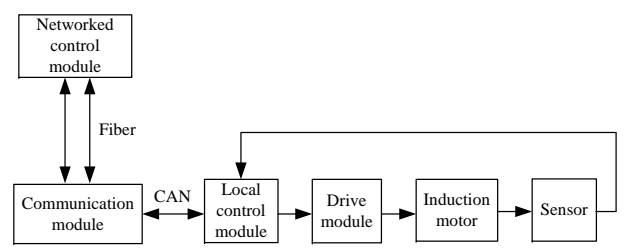

Fig. 8. Structure of the NMCS experiment platform

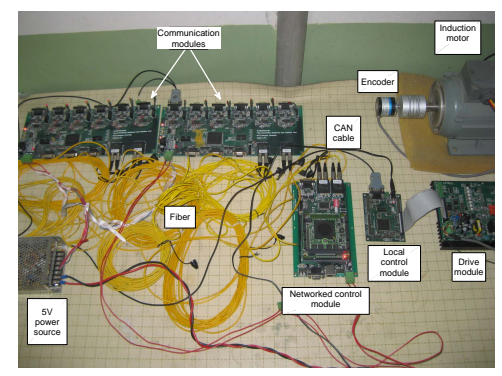

Fig. 9. NMCS experiment platform

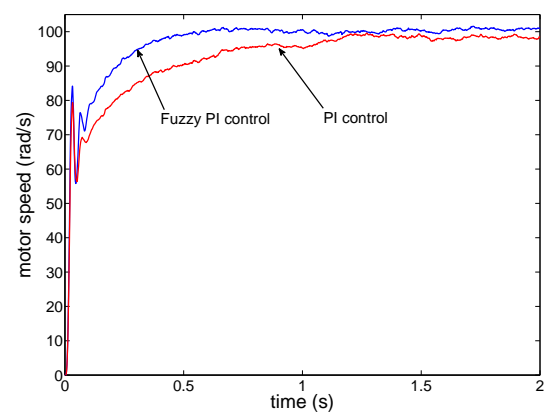

Fig. 10. Experimental results using different networked controllers

The communication module is employed to transform the signals between the two spatially distributed parts with different communication protocols. The signals transmission between the networked control module and the communication module is fulfilled by the fiber with high rate. The CAN cable is used in data transmission between the local control system and the communication module, which is widely used in modern industrial applications. It is supposed that no load on the motor. The real NMCS experiment platform is shown in Fig. 9, and the rated parameters of the induction motor is listed in Table III. The experimental results are illustrated in Fig. 10, which show that the proposed control method hold faster response than the conventional PI control.

\section{CONCLUSIONS}

This paper extends the application areas of NMCSs into nonlinear induction motors field. A hierarchical modeling approach is proposed, such that the simplified linearized induction motor model can be adopted in NMCSs. The fuzzy gain scheduling of the networked speed controller enables flexibility against the QoS variation in the network. A state predictor is designed in the feedback channel. Simulation and experimental results showed the effectiveness of the proposed approach. There are several aspects to be investigated in the future: (1) more reasonable modeling and control methods in applications; (2) networked position control of induction motors; and (3) rational ways in selecting controller gains.

\section{REFERENCES}

[1] C. L. Hwang, L. J. Chang, and Y. S. Yu, "Network-based fuzzy decentralized sliding-mode control for car-like mobile robots," IEEE Transactions on Industrial Electronics, vol. 54, no. 1, pp. 574-585, 2007.

[2] N. Chopra, P. Berestesky, and M. W. Spong, "Bilateral teleoperation over unreliable communication networks," IEEE Transactions on Control Systems Technology, vol. 16, no. 2, pp. 304-313, 2008.

[3] K. Natori, T. Tsuji, K. Ohnishi, A. Hace, and K. Jezernik, "Timedelay compensation by communication disturbance observer for bilateral teleoperation under time-varying delay," IEEE Transactions on Industrial Electronics, vol. 57, no. 3, pp. 1050-1062, 2010.

[4] K. B. Purvis, K. J. Astrom, and M. Khammash, "Estimation and optimal configurations for localization using cooperative UAVs," IEEE Transactions on Control Systems Technology, vol. 16, no. 5, pp. 947958, 2008.

[5] J. Tisdale, Z. Kim, and J. K. Hedrick, "Autonomous UAV path planning and estimation an online path planning framework for cooperative search and localization," IEEE Robotics \& Automation Magazine, vol. 16, no. 2, pp. 35-42, 2009.

[6] I. Maza, K. Kondak, M. Bernard, and A. Ollero, "Multi-UAV cooperation and control for load transportation and deployment," Journal of Intelligent \& Robotic Systems, vol. 57, no. 4, pp. 417-449, 2010.

[7] J. Marescaux, J. Leroy, M. Gagner, F. Rubino, D. Mutter, M. Vix, S. E. Butner, and M. K. Smith, "Transatlantic robot-assisted telesurgery," Nature, vol. 413, no. 6854, pp. 379-380, 2001.

[8] S. Rothenberg, G. Holcomb, K. Georgeson, M. Irish, E. Lucas, and T. Blinman, "Web-based live telesurgery for minimally invasive procedures in children as an educational tool," Journal of Laparoendoscopic \& Advanced Surgical Techniques, vol. 17, no. 2, pp. 226-229, 2007.

[9] H. H. King, K. Tadano, R. Donlin, D. Friedman, M. J. H. Lum, V. Asch, C. Wang, K. Kawashima, and B. Hannaford, "Preliminary protocol for interoperable telesurgery," in the $14^{\text {th }}$ International Conference on Advanced Robotics, 2009, pp. 680-685.

[10] B. K. Kim, W. K. Chung, and I. H. Suh, "Robust synchronizing motion control of twin-servo systems based on network modeling," in the $39^{\text {th }}$ IEEE Conference on Decision and Control, 2000, pp. 1019-1024.

[11] H. B. Li, M. Y. Chow, and Z. Q. Sun, "EDA-based speed control of a networked dc motor system with time delays and packet losses," IEEE Transactions on Industrial Electronics, vol. 56, no. 5, pp. 1727-1735, 2009.

[12] L. Samaranayake, "Distributed control of electric drives via Ethernet," Ph.D. dissertation, Royal Institute of Technology, Stockholm, Sweden, 2003.

[13] H. Zhang, Y. Shi, and A. S. Mehr, "Robust static output feedback control and remote PID design for networked motor systems," IEEE Transactions on Industrial Electronics, vol. 58, no. 12, pp. 5396-5405, 2011.

[14] B. K. Bose, Modern Power Electronics and AC Drives. New Jersey, USA: Prentice Hall, 2002.

[15] Z. Yan and V. Utkin, "Sliding mode observers for electric machines - An overview," in Proceedings of the 28th Annual Conference of the IEEE Industrial Electronics Society, 2002, pp. 1842-1847.

[16] P. Park, Y. S. Moon, and W. H. Kwon, "A delay-dependent robust stability criterion for uncertain time-delay systems," in the 1998 American Control Conference, 1998, pp. 1963-1964.

[17] S. Boyd, L. E. Ghaoui, E. Feron, and V. Balakrishnan, Linear Matrix Inequalities in System and Control Theory. Philadelphia, USA: PA of SIAM, 1994.

[18] A. Hassibi, S. P. Boyd, and J. P. How, "Control of asynchronous dynamical systems with rate constraints on events," in the $38^{\text {th }}$ IEEE Conference on Decision and Control, 1999, pp. 1345-1351.

[19] A. Rabello and A. Bhaya, "Stability of asynchronous dynamical systems with rates constraints and applications," IEE ProceedingsControl Theory and Applications, vol. 150, no. 5, pp. 546-550, 2003. 